\title{
Atuação fisioterapêutica na esclerose tuberosa associada a linfangioleiomiomatose
}

\author{
Physiotherapeutic performance in tuberous sclerosis associated with lymphangioleiomyomatosis \\ Actuación fisioterapéutica en la esclerosis tuberosa asociada a linfangioleiomiomatosis
}

Recebido: 28/01/2022 | Revisado: 01/02/2022 | Aceito: 03/02/2022 | Publicado: 05/02/2022

\author{
Caio Fernandes de Almeida \\ ORCID: https://orcid.org/0000-0001-9108-5799 \\ Hospital Universitário do Oeste do Paraná, Brasil \\ E-mail: caiofernandes66@gmail.com \\ Juliana Hering Genske \\ ORCID: https://orcid.org/0000-0001-6896-0903 \\ Hospital Universitário do Oeste do Paraná, Brasil \\ E-mail: juliana.hering@ terra.com.br \\ Fernanda da Silva Tori \\ ORCID: https://orcid.org/0000-0002-5140-3892 \\ Hospital Universitário do Oeste do Paraná, Brasil \\ E-mail: ferdstori@gmail.com \\ Juliana Almeida Burgarelli \\ ORCID: https://orcid.org/0000-0002-2559-1914 \\ Hospital Universitário do Oeste do Paraná, Brasil \\ E-mail: Burgarellijuliana@gmail.com \\ Alessandro Schaefer \\ ORCID: https://orcid.org/0000-0002-3647-5118 \\ Hospital Universitário do Oeste do Paraná, Brasil \\ E-mail: alessandroschaefer@hotmail.com \\ Janaina Abramovecht \\ ORCID: https://orcid.org/0000-0001-8443-6976 \\ Hospital Universitário do Oeste do Paraná, Brasil \\ E-mail: janaina_abramovecht47@hotmail.com \\ Jessica Magnante \\ ORCID: https://orcid.org/0000-0002-5974-3517 \\ Hospital Universitário do Oeste do Paraná, Brasil \\ E-mail: jehmagnante97@gmail.com \\ Daiane Maria Pastorio \\ ORCID: https://orcid.org/0000-0001-5813-478X \\ Hospital Universitário do Oeste do Paraná, Brasil \\ E-mail: Daiane.adp.18@outlook.com
}

\begin{abstract}
Resumo
A esclerose tuberosa é uma doença rara, de caráter genético e degenerativo, onde ocorre anomalias no genes TSC1 ou TSC2, dos cromossomos 9 e 16, secundariamente ao processo fisiopatológico dessa doença pode ocorrer a linfangioleiomiomatose pulmonar (LAM), uma neoplasia de baixo grau caracterizada pela proliferação de células musculares lisas no parênquima pulmonar, podendo ocasionar diversas manifestações clínicas, tais como: pneumotórax espontâneo e recorrente, dispneia, tosse e redução da capacidade funcional do indivíduo. Desta forma os pacientes possuem uma evolução clínica para deterioração das capacidades pulmonares e distúrbio da homeostase respiratória. Mesmo com o avanço da medicina ainda não existe uma cura para essas doenças. Neste estudo, relata-se um caso de uma paciente adulta, do sexo feminino, com diagnóstico de esclerose tuberosa e linfangioleiomiomatose pulmonar, tratada no Hospital Universitário do Oeste do Paraná (HUOP), portanto, este relato descreve abordagens fisioterapêuticas aplicadas em diferentes momentos da hospitalização em uma paciente portadora de esclerose tuberosa e linfangioleiomiomatose e assim podendo correlacionar com os possíveis benefícios da intervenção e tratamento fisioterapêutico. Paciente evoluiu para alta melhorada, ativa e sem necessidade de oxigenoterapia. Concluise que a fisioterapia é uma importante aliada no auxílio do tratamento da esclerose tuberosa e linfangioleiomiomatose. Palavras-chave: Esclerose tuberosa; Linfangioleiomiomatose; Serviço hospitalar de fisioterapia; Reabilitação.
\end{abstract}

\begin{abstract}
Tuberous sclerosis is a rare disease, of genetic and degenerative character, where there are anomalies in the TSC1 or TSC2 genes, of chromosomes 9 and 16. Secondary to the pathophysiological process of this disease, pulmonary lymphangioleiomyomatosis (LAM) can occur, a low-grade neoplasm characterized by the proliferation of smooth muscle cells in the lung parenchyma, which can cause several clinical manifestations, such as: spontaneous and recurrent pneumothorax, dyspnea, cough and reduced functional capacity of the individual. Thus, patients have a
\end{abstract}


clinical evolution towards deterioration of lung capacity and respiratory homeostasis disorder.Even with the advancement of medicine, there is still no cure for these diseases. In this study, we report a case of an adult female patient, diagnosed with tuberous sclerosis and pulmonary lymphangioleiomyomatosis, treated at the university hospital in western paraná (HUOP). hospitalization in a patient with tuberous sclerosis and lymphangioleiomyomatosis and thus correlate with the possible benefits of intervention and physical therapy treatment. Patient evolved to discharge improved, active and without the need for oxygen therapy. Therefore, it is concluded that physical therapy is an important ally in helping to treat tuberous sclerosis and lymphangioleiomyomatosis.

Keywords: Tuberous Sclerosis; Lymphangioleiomyomatosis; Physiotherapy hospital service; Rehabilitation.

\section{Resumen}

La esclerosis tuberosa es una enfermedad rara, de carácter genético y degenerativo, donde existen anomalías en los genes TSC1 o TSC2, de los cromosomas 9 y 16. Secundaria al proceso fisiopatológico de esta enfermedad, puede ocurrir linfangioleiomiomatosis pulmonar (LAM), una baja neoplasia de grado caracterizada por la proliferación de células de músculo liso en el parénquima pulmonar, que puede ocasionar diversas manifestaciones clínicas, tales como: neumotórax espontáneo y recurrente, disnea, tos y disminución de la capacidad funcional del indivíduo. Así, los pacientes tienen una evolución clínica hacia el deterioro de la capacidad pulmonar y el trastorno de la homeostasis respiratoria. Incluso con el avance de la medicina, todavía no existe cura para estas enfermedades. En este estudio se reporta el caso de una paciente adulta, diagnosticada de esclerosis tuberosa y linfangioleiomiomatosis pulmonar, atendida en el hospital universitario de paraná occidental (HUOP). Hospitalización en un paciente con esclerosis tuberosa y linfangioleiomiomatosis y así poder correlacionar con los posibles beneficios de la intervención y el tratamiento de fisioterapia. El paciente evolucionó hasta el alta mejorada, activa y sin necesidad de oxigenoterapia. Se concluye que la fisioterapia es un aliado importante para ayudar a tratar la esclerosis tuberosa y la linfangioleiomiomatosis.

Palabras clave: Esclerosis tuberosa; Linfangioleiomiomatosis; Servicio hospitalario de fisioterapia; Rehabilitación.

\section{Introdução}

A Esclerose Tuberosa (ET) é uma patologia rara com baixa incidência 1:10000, possui caráter genético, de herança autossômica dominante, onde ocorre anomalias nos genes TSC1 ou TSC2, dos cromossomos 9 e 16, caracterizada por lesões neoplásicas benignas com manifestações clínicas multissistêmicas, podendo estar associada a linfangioleiomiomatose pulmonar (Reis Filho et al., 1998)

A linfangioleiomiomatose (LAM) é uma enfermidade rara, com incidência de cerca de 1/1.000.000, com predominância em mulheres adultas, causando proliferação de células musculares anormais no parênquima pulmonar e linfonodos. Sua manifestação clínica se dá através de dispneia progressiva, pneumotórax de repetição e tosse seca (Medeiros Junior \& Roberto Ribeiro Carvalho, 2004)

O paciente acometido por esses processos fisiopatológicos, apresentam deterioração e diminuição contínua da função pulmonar, podendo alterar o volume expiratório forçado no primeiro segundo (VEF1), redução da capacidade vital e aumento gradual do volume residual (Medeiros Junior \& Roberto Ribeiro Carvalho, 2004). Além das alterações supracitadas podem ocorrer situações de emergência como pneumotórax de repetição, onde se faz necessário a abordagem cirúrgica para drenagem de tórax e em alguns casos até mesmo a pleurodese química.

Os achados nos exames de imagem encontrados em pacientes portadores da LAM, associam-se a existência de hiperinsuflação pulmonar, infiltrado intersticial reticulonodular e cistos de diferentes tamanhos em topografia pulmonar. Podendo caracterizar um distúrbio ventilatório obstrutivo (Roberto Agea Cutolo et al., 2018).

Atualmente o tratamento da Linfangioleiomiomatose baseia-se em solucionar as situações de urgência, como: pneumotórax de repetição, quilotórax e ascite quilosa, onde utiliza-se a drenagem torácica através da inserção de um dreno no tórax. Também é realizado o tratamento anti-hormonal como uma tentativa de inibir e suprimir da secreção estrogênica (Medeiros Junior \& Roberto Ribeiro Carvalho, 2004). Em 2017 foi aprovado pelo Sistema Único de Saúde (SUS) a utilização 
do medicamento Sirolimo (medicamento de alto custo) para tratamento da linfangioleiomiomatose (Associação dos Portadores de Linfangioleiomiomatose no Brasil- ALAMBRA, 2017). O uso desse medicamento vem demonstrando respostas favoráveis nas manifestações clínicas dos pacientes, atuando na inibição da MTOR (Mammalian Target of Rapamycin), uma proteína que atua na síntese com papel principal no crescimento, proliferação e na manutenção das células (Freitas et al., 2015).

A eficácia e importância da fisioterapia respiratória durante a internação está mais do que comprovada na literatura, considerando que diminuí o tempo de internação, complicações, custos hospitalares e reabilita o paciente para a fase pósinternação (Nunes Alvez, 2012). Portanto vale salientar a importância e abordagem fisioterapêutica, desta forma contribuindo para a comunidade científica com dados sobre os pacientes portadores de esclerose tuberosa e linfangioleiomiomatose.

\section{Metodologia}

O presente artigo trata-se de um relato de caso (Vieira \& Hossne, 2021), ocorrido no Hospital Universitário do Oeste do Paraná, feito através de análise de prontuário eletrônico (Software TASY), prontuário físico e exames complementares, todos encontrados nas dependências do hospital. Desta forma, foram analisados sinais e sintomas, processo fisiopatológico da doença e complicações durante internação, tratamento realizado pela equipe fisioterapêutica, multidisciplinar e informações coletadas na alta hospitalar.

Este relato de caso teve aprovação do Comitê de Ética em Pesquisa e utilização do Termo de Consentimento Livre e Esclarecido, onde ambos foram assinados pela paciente.

\section{Relato do Caso}

Paciente do sexo feminino, 43 anos, brasileira e agricultora, admitida no pronto-socorro do Hospital Universitário do Oeste do Paraná no dia 31/08/2021, Glasgow 15, estável hemodinamicamente, em ar ambiente, com queixa de dispneia e hipossaturação ( $\mathrm{SpO} 2$ 87-88\%), com ausculta pulmonar apresentando murmúrios vesiculares audíveis, porém reduzidos em base esquerda. Apresentava dreno de tórax fechado (DTF) em selo d'água (oscilante, borbulhante e com débito sanguinolento), inserido pela equipe de transporte aéreo devido a pneumotórax em hemitórax esquerdo (HTE). Paciente possuí diagnóstico prévio de esclerose tuberosa associada a linfangioleiomiomatose. Como histórico pregresso, realizou uma nefrectomia em 2014 por angiomiolipomas, hernioplastia umbilical em 2016 e seu histórico familiar apresenta uma avó portadora de esclerose tuberosa. Após estabilização da paciente foi ofertado oxigenoterapia em baixo fluxo, realizado exames de imagens e laboratoriais, onde confirmou alterações tomográficas (Figura 1) e uma leucocitose (20.720/mm3) visualizada através do hemograma de admissão. 
Figura 1: Tomografia de tórax, corte coronal de janela pulmonar.

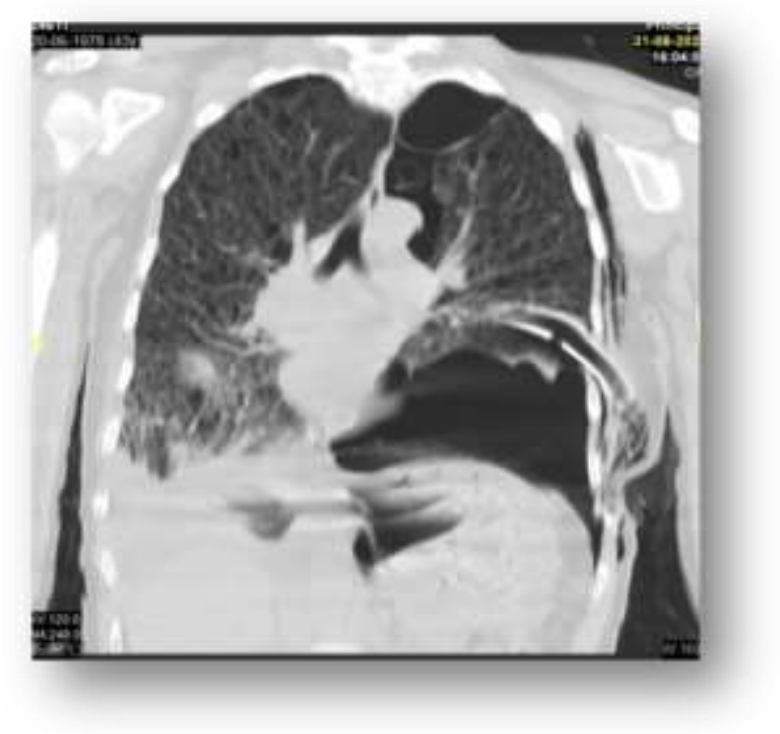

Fonte: Autores (2022).

$\mathrm{Na}$ imagem de tomografia de tórax observa-se diversos cistos bilaterais de paredes finas de tamanho uniforme, pneumotórax a esquerda e uso de dreno de tórax (DTF), derrame pleural em base pulmonar direita e enfisema subcutâneo torácico no lado esquerdo. Sugerindo alterações parenquimatosas e restritivas.

O tratamento fisioterapêutico foi ofertado durante toda internação, da data de entrada 31/08/2021 até alta hospitalar 13/10/2021. Na fase inicial os atendimentos ocorriam durante 02 vezes ao dia, e na fase intermediária e final com uma frequência de 01 vez ao dia. Inicialmente foi realizado uma avaliação fisioterapêutica hospitalar onde foram verificados, sinais vitais, capacidade de exercício e mobilidade no leito, capacidade de tosse, nível de cooperação, tipo de oxigenoterapia ofertada e adequação da oxigenoterapia. Posteriormente as condutas fisioterapêuticas na fase inicial foram focadas na reexpansão pulmonar tendo em vista a necessidade da utilização do dreno de tórax. Todas as fases contendo condutas e objetivos foram descritas conforme a Tabela 1 . 
Tabela 1: Correlacionando as fases de internação, condutas e objetivos.

\begin{tabular}{|c|c|c|}
\hline & CONDUTAS & OBJETIVOS \\
\hline FASE INICIAL & $\begin{array}{l}\text {-Padrões respiratórios } \\
\text {-Reexpansão por pressão positiva } \\
\text { intermitente com baixa pressão } \\
\text {-Deambulação curtas distâncias } \\
\text {-Oxigenoterapia em baixo fluxo }\end{array}$ & $\begin{array}{l}\text {-Reexpansão pulmonar } \\
\text {-Resolutividade da necessidade do dreno de tórax. } \\
\text {-Manutenção da capacidade funcional }\end{array}$ \\
\hline $\begin{array}{l}\text { FASE } \\
\text { INTERMEDIÁRIA }\end{array}$ & $\begin{array}{l}\text {-Padrões respiratórios } \\
\text {-Estímulo de tosse (produtiva) } \\
\text {-Incentivador (Threshold® PEP) } \\
\text {-Deambulação moderadas distâncias } \\
\text {-Oxigenoterapia em baixo fluxo }\end{array}$ & $\begin{array}{l}\text {-Reexpansão pulmonar } \\
\text {-Resolutividade da necessidade do dreno de tórax } \\
\text {-Recuperação da capacidade funcional } \\
\text {-Redução da necessidade de } \mathrm{O} 2 \text { suplementar }\end{array}$ \\
\hline FASE FINAL & $\begin{array}{l}\text {-Padrões respiratórios } \\
\text {-Estímulo de tosse (produtiva) } \\
\left.\text {-Incentivador (Respiron }{ }^{\circledR}+\text { Shaker }{ }^{\circledR}\right) \\
\text {-Deambulação grandes distâncias } \\
\text {-Oxigenoterapia em baixo fluxo }\end{array}$ & $\begin{array}{l}\text {-Melhorar alterações obstrutivas causadas pelo } \\
\text { processo fisiopatológico da doença } \\
\text {-Desmame da oxigenoterapia } \\
\text {-Melhora da capacidade funcional }\end{array}$ \\
\hline
\end{tabular}

Fonte: Autores (2022).

Durante as fases de atendimento ocorreram intercorrências clínicas, tais como: piora da função renal, necessidade de abordagem cirúrgica e perda do dreno de tórax, todas essas alterações estão descritas conforme Tabela 2 com suas respectivas correlações e datas.

Tabela 2: Datas e complicações durante período de hospitalização.

INTERCORRÊNCIAS

\begin{tabular}{|c|c|}
\hline PIORA DA FUNCÃO RENAL & 07/09/2021 \\
NECESSIDADE DE PLEURODESE & A \\
PERDA DO DRENO DE TÓRAX & 21 \\
21/09/2021 &
\end{tabular}

PIORA DA FUNCÃO RENAL

$07 / 09 / 202$

\section{CONDUTAS MÉDICAS}

Otimização da hidratação via endovenosa

Abordagem cirúrgica de decorticação pulmonar

Recolocação de novo DTF

\section{DESFECHOS}

Melhora do quadro

Não realizado pleurodeses por difícil indentificação da janela pulmonar

DTF eficiente

Fonte: Autores (2022).

$\mathrm{Na}$ fase final da intervenção fisioterapêutica, a paciente apresentava boa evolução clínica, o dreno de tórax foi retirado no dia 07/10/2021 pela equipe cirúrgica, após resolutividade do pneumotórax (Figura 2). A paciente encontrava-se sem necessidade de oxigenoterapia suplementar, deambulando moderadas distâncias, com significativa redução na produtividade de secreção pulmonar, relatando melhora da dispneia e sem apresentar novos quadros de hipossaturação. Paciente adquiriu alta hospitalar no dia 13/10/2021 com indicação de acompanhamento multidisciplinar a nível ambulatorial e através dos recursos disponibilizados pelo Sistema Único de Saúde (SUS). 
Figura 2: Raio-X frontal de tórax.

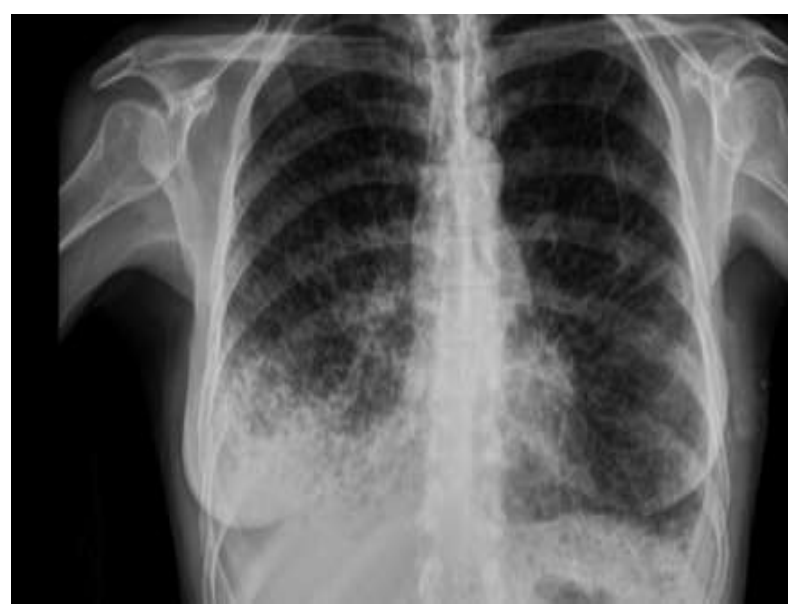

Fonte: Autores (2022).

Conforme figura superior, visualiza-se pequenos cistos bilaterais, com consolidação focal em seio costofrênico direto, sugerindo processo patológico de derrame pleural e alteração pulmonar cística.

\section{Discussão}

Autores trazem a linfangioleiomiomatose como uma fisiopatologia com características de um distúrbio ventilatório obstrutivo (Roberto Agea Cutolo et al., 2018) porém o pneumotórax espontâneo e o uso do dreno de tórax, enquadravam a paciente em uma alteração restritiva no momento da internação, fazendo-se necessário o uso de recursos para reexpansão pulmonar (Evangelista et al., 2019).

Os casos de pneumotórax espontâneo secundários a Linfangioleiomiomatose, semelhante ao caso supracitado, são citados por autores como uma das manifestações clínicas recorrentes, podendo aumentar a morbimortalidade em pacientes portadores de LAM (Grigorescu et al., 2007). Segundo Riojas (2012), a LAM era difícil de diagnosticar e tinha uma mortalidade de $100 \%$ após 10 anos, porém atualmente existe uma sobrevida de $71 \%$ após 10 anos.

O processo fisiopatológico pulmonar ocorre em concomitância a linfangioleiomiomatose, gerada pela substituição do tecido alveolar fisiológico por cistos e músculo liso (Freitas et al., 2015), levando a alterações vasculares e brônquicas, desta forma, desencadeando a sintomatologia respiratória.

$\mathrm{O}$ angiomiolipoma renal é uma patologia normalmente secundária à esclerose tuberosa, ocasionando a presença de diversos tumores benignos, de crescimento progressivo levando ao acometimento da função renal (Bissler et al., 2013). Portanto, com a cirurgia prévia da paciente, onde foi realizado uma nefrectomia pela presença de angiomiolipomas, pode ter uma correlação com sua piora da função renal durante o internamento.

O diagnóstico da linfangioleiomiomatose (LAM) é baseado em achados na tomografia computadorizada de alta resolução (TCAR), onde deve correlacionar achados característicos, como cistos pulmonares difusos de paredes regulares e finas, associado a derrame pleural/ascite quilosa, linfangioleiomioma/envolvimento ganglionar. A biópsia do tecido pulmonar também pode servir como guia para diagnóstico (Medeiros Junior \& Roberto Ribeiro Carvalho, 2004)

Alguns autores caracterizam que a linfangioleiomiomatose no seu estado mais avançado levam aos seus portadores uma obstrução irreversível do fluxo aéreo (Yen et al., 2005). Desta forma levando a alterações expressivas no volume expiratório forçado no primeiro segundo $\left(\mathrm{VEF}_{1}\right)$ e na capacidade vital forçada $(\mathrm{CVF})$.

$\mathrm{Na}$ fase inicial do tratamento foi utilizado a respiração por pressão positiva intermitente (RPPI) com baixa pressão $\left(8 \mathrm{cmH}_{2} \mathrm{O}\right)$ para auxílio da reexpansão pulmonar no lado acometido pelo pneumotórax e com dreno de tórax. Na ocasião, o 
mesmo encontrava-se oscilante, borbulhante e com pequeno débito sanguinolento. Porém, 3 dias após, houve a necessidade de suspensão da terapia com pressão positiva pelo aumento circunstancial do enfisema subcutâneo. Alguns pesquisadores reforçam o uso do RPPI como uma forma rápida e fácil acesso como técnica reexpansiva e pode ser indicado para tórax drenados (da Conceição dos Santos \& Lunardi, 2015). Estudos apontam como uma possível complicação do uso da pressão positiva intermitente a possibilidade da presença ou aumento do enfisema subcutâneo (Pasini, 2004).

As técnicas e recursos mais utilizadas na reabilitação de pacientes portadores de dreno de tórax, são: uso de incentivadores respiratórios, manobras de reexpansão pulmonar, deambulação, tosse cinética, cinesioterapia e posicionamento funcional (Duarte et al., 2020). Esses recursos foram utilizados em diferentes fases de reabilitação da paciente (conforme a tabela 1), contribuindo para melhora da função pulmonar da mesma.

O pronto-socorro e emergência são considerados caminhos de acessos para os pacientes críticos, com objetivo de minimizar a mortalidade dos pacientes, onde a fisioterapia é uma profissão de primeiro contato, podendo atuar na prevenção e tratamento do paciente crítico (Silva \& Santos, 2019). A fisioterapia no âmbito hospitalar proporciona ao paciente crítico, diminuição da sintomatologia, recuperação da capacidade funcional e qualidade de vida, além de auxiliar na redução do tempo de internação hospitalar e diminuição dos custos do tratamento (cunha et al., 2009).

O uso da drenagem de tórax em selo d'água é um fator correlacionado com a presença de dor e limitação funcional (Pereira De Lima et al., 2008). Alguns autores acentuam que deambular mais precocemente e sair do leito pode influenciar diretamente na melhora da capacidade funcional (Nomori et al., 2001).

Segundo o Conselho Regional de Enfermagem do estado de São Paulo (COREN/SP), a perda do dreno de tórax é uma complicação recorrente, podendo aumentar a morbidade do paciente, prolongar o tempo de hospitalização, favorecer o surgimento de novas complicações pulmonares tais como: pneumotórax hipertensivo, enfisema subcutâneo e hemotórax.

As complicações clínicas podem influenciar diretamente no tempo de internação hospitalar, estando correlacionadas diretamente com taxa de morbimortalidade. Estudos cardiológicos apontam a insuficiência renal como uma das principias complicações, desta forma pode estar correlacionada diretamente com o risco de morte do paciente (Carvalho et al., 2011)

\section{Conclusão}

Conclui-se que o caso da paciente teve um desfecho satisfatório e que a atuação da fisioterapia no âmbito hospitalar proporciona um importante papel complementar no tratamento dos pacientes portadores de esclerose tuberosa e linfangioleiomiomatose, desta forma, atuando na reabilitação pulmonar, na capacidade funcional, redução das chances de surgimento de novas complicações pulmonares e osteomioarticulares.

Possíveis sugestões para trabalhos futuros, onde avalia-se pacientes portadores de esclerose tuberosa e linfangioleiomiomatose com acometimento pulmonar, seria, uma abordagem quantitativa com dados sobre capacidades pulmonares, função pulmonar, força muscular inspiratória e capacidade de exercício, incluindo no âmbito pré-operatório e pósoperatório nos pacientes que precisam de uma abordagem invasiva.

\section{Referências}

Alshoabi, S. A., Hamid, A. M., Alhazmi, F. H., Qurashi, A. A., Abdulaal, O. M., Aloufi, K. M., \& Daqqaq, T. S. (2022). Diagnostic features of tuberous sclerosis complex: case report and literature review. Quantitative Imaging in Medicine and Surgery, 12(1), 846.

Associação dos portadores de Linfangionleiomiomatose no Brasil - ALAMBRA. (2017). Recuperado de https://www.alambrabrasil.org/

Bissler, J. J., Kingswood, J. C., Radzikowska, E., Zonnenberg, B. A., Frost, M., Belousova, E., \& Budde, K. (2013). Everolimus for angiomyolipoma associated with tuberous sclerosis complex or sporadic lymphangioleiomyomatosis (EXIST-2): a multicentre, randomised, double-blind, placebo-controlled trial. The Lancet, 381(9869), 817-824. 
Carvalho, M. R. M., Silva, N. A. S., Oliveira, G. M. M., \& Klein, C. H. (2011). Complicações e tempo de internação na revascularização miocárdica em hospitais públicos no Rio de Janeiro. Revista Brasileira de Terapia Intensiva, 23(3), 312-320.

Carneiro, A. S., Mafort, T. T., \& Lopes, A. J. (2021). A 34-Year-Old Woman from Brazil with Pulmonary Lymphangioleiomyomatosis Diagnosed by Raised Serum Vascular Endothelial Growth Factor-D (VEGF-D) Levels and Lung Cysts on Computed Tomography Imaging Presenting with COVID-19 Pneumonia. The American Journal of Case Reports, 22, e932660-1.

Conselho Regional de Enfermagem de São Paulo. (2011). Documentos básicos de enfermagem: enfermeiros, técnicos, auxiliares. Boas práticas Dreno de tórax. Recuperado de https://portal.coren-sp.gov.br/sites/default/files/dreno-de-torax.pdf

Cunha., C. S., Soares, B., \& Nascimento, R. R. (2009). Técnicas reexpansivas no derrame pleural - uma revisão de literatura. Cadernos UniFOA, 4(9), 63-67.

Santos, E. C., \& Lunardi, A. C. (2015). Efficacy of the addition of positive airway pressure to conventional chest physiotherapy in resolution of pleural effusion after drainage: protocol for a randomised controlled trial. Journal of Physiotherapy, 61(2), 93.

Duarte, M. P., Rosa, L. L. D., Pinheiro, E. M., Alves, I. K., Santos, A. A., Andrade, Á. D. B., \& Diniz, W. A. (2020). Influência da fisioterapia na reabilitação de pacientes submetidos à drenagem torácica em um hospital de urgência e emergência da Amazônia legal. Revista Eletrônica Acervo Saúde, (45), e2959e2959.

Evangelista, A. R., Morais, R. A., Moura, F. M., Carvalho, K. M. M. B. (2019). Intervenções fisioterapêuticas no tratamento e estabilização de pacientes com pneumotórax. Encontro de extensão, docência e iniciação científica (eedic), 5(1).

Freitas, C. S. G., Baldi, B. G., Araújo, M. S., Heiden, G. I., Kairalla, R. A., \& Carvalho, C. R. R. (2015). Use of sirolimus in the treatment of lymphangioleiomyomatosis: favorable responses in patients with different extrapulmonary manifestations. Jornal Brasileiro de Pneumologia, 41(3), 275-280.

Grigorescu C., Bosânceanu M., Boişteanu D., Aldea A., Chiseliţă I., Cozma L.G. (2007). Limfangioleiomiomatoza cauză rară de pneumotorax spontan bilateral [Lymphangioleiomyomatosis (LAM)--an uncommon cause of bilateral spontaneous pneumothorax]. Revista Medico-chirurgicala a Societatii de Medici si Naturalist Din Iasi, 111(1), 125-128.

Medeiros, P. J., \& Carvalho, C. R. R. (2004). Linfangioleiomiomatose pulmonar. Jornal Brasileiro de Pneumologia, 30(1), 66-77.

Nomori, H., Horio, H., Suemasu, K. (2001). Early removal of chest drainage tubes and oxygen support after a lobectomy for lung cancer facilitates earlier recovery of the 6-minute walking distance. Surgery Today, 31, 395-399.

Alvez, A. N. (2012). A importância da atuação do fisioterapeuta no ambiente hospitalar. Ensaios e Ciência: Ciências Biológicas, Agrárias e Da Saúde, 16(6), $173-184$.

Pasini, R. (2004). Complicação relacionada ao uso da respiração com pressão positiva intermitente. Fisioterapia Brasil, 5(3), $242-245$.

Lima, V. P., Bonfim, D., Risso, T. T., Paisani, D. M., Fiore, J. F. J., Chiavegato, L. D., \& Faresin, S. M. (2008). Influência do dreno pleural sobre a dor, capacidade vital e teste de caminhada de seis minutos em pacientes submetidos à ressecção pulmonar. In Jornal Brasileiro de Pneumologia, 34(12), 10031007

Reis, J. S. F., Netto, M. H. M., Loyola, J. G. N., Araújo, J. C., Antoniuk, S., \& Torres, L. F. B. (1998). Eclerose tuberosa: relato de caso com estudo histopatológico e ultraestrutural. Arquivos de Neuro-Psiquiatria, 56(3 B), 671-676.

Riojas RA., Bahr BA., Thomas DB, Perciballi J., Noyes L. (2012) A case report of lymphangioleiomyomatosis presenting as spontaneous pneumothorax. Mil Med,177(4):477-80.

Cutolo, L. R. A., Bonassa, R. T., Stahelin, A. H. S., \& Macedo, L. S. (2018). linfangioleiomiomatose pulmonar em paciente pediátrico: relato de caso. Arquivos Catarinenses de Medicina, 47(1), 216-223.

Silva, C. C. M., \& Santos, I. M. (2019). A importância da fisioterapia no setor de urgência e emergência: uma revisão de literatura. Brazilian Journal of Development, 5(10), 18335-18343.

Vieira, S., \& Hossne, W. S. (2021). Metodologia científica para a área da saúde (3a ed.). Guanabara Koogan.

Yen, K. T., Putzke, J. D., Staats, B. A., \& Burguer, C. D. (2005). The prevalence of acute response to bronchodilator in pulmonary lymphangioleiomyomatosis. Respirology, 10(5), 643-648. 